\title{
Analysis of Combustion Characteristics when Adding Hydrogen and Short Chain Hydrocarbons to RP-3 Aviation Kerosene Based on Variation Disturbance Method
}

Shuhao Li $i^{\dagger}$, Junjiang Guo ${ }^{\ddagger}$,Zhenghe Wang ${ }^{\dagger}$, Shuanghui Xi ${ }^{\S}$, Junxing Hou ${ }^{\dagger}, Z_{\text {Zhenhua Wen }}{ }^{\dagger, *}$

†School of Aeronautical Engineering, Zhengzhou University of Aeronautics, Zhengzhou 450046

, People's Republic of China.

¥School of Chemical Engineering, Guizhou Institute of Technology, Guiyang 550003, People’s Republic of China.

${ }^{\S}$ School of Chemical Engineering, Sichuan University, Chengdu 610065, People’s Republic of China.

\section{Numerical simulation}

A numerical study is performed by Chemkin software ${ }^{1}$. The simulation are set out as follows: ignition delay time, adiabatic flame temperature, burnout time and rate of production are conducted in a Closed Homogeneous Batch Reactor. Meanwhile the extinction temperature is performed via Perfectly Stirred Reactor. Laminar flame speed is calculated by Premixed Laminar Flame Speed Calculation code. Extinction strain rate is simulated with the help of the Diffusion Opposed-flow Flame code and the strain rate is defined on the oxidizer side as the gradient of the velocity profile ${ }^{2}$. 


\section{DETAILED RESULTS of RP-3 and BLENDED FUELS}

Table 1S. Ignition delay time of different fuels, unit: s.

\begin{tabular}{|c|c|c|c|c|c|c|c|c|}
\hline $\begin{array}{c}\text { Pressure } \\
(\mathbf{M P a})\end{array}$ & $\begin{array}{c}\text { Temperature } \\
\mathbf{( K )}\end{array}$ & $\mathbf{R P - 3}$ & $\mathbf{B F - 1}$ & $\mathbf{B F - 2}$ & $\mathbf{B F - 3}$ & $\mathbf{B F}-4$ & BF-5 & BF-6 \\
\hline \multirow{5}{*}{0.1} & 1000 & $1.6575 \mathrm{E}-02$ & $1.6489 \mathrm{E}-02$ & $1.6727 \mathrm{E}-02$ & $1.6628 \mathrm{E}-02$ & $1.6793 \mathrm{E}-02$ & $1.6682 \mathrm{E}-02$ & $1.7205 \mathrm{E}-02$ \\
\cline { 2 - 9 } & 1200 & $7.6383 \mathrm{E}-04$ & $7.5306 \mathrm{E}-04$ & $7.7887 \mathrm{E}-04$ & $7.2360 \mathrm{E}-04$ & $7.5385 \mathrm{E}-04$ & $8.2963 \mathrm{E}-04$ & $8.0772 \mathrm{E}-04$ \\
\cline { 2 - 9 } & 1400 & $1.1074 \mathrm{E}-04$ & $1.0859 \mathrm{E}-04$ & $1.1325 \mathrm{E}-04$ & $1.0466 \mathrm{E}-04$ & $1.0742 \mathrm{E}-04$ & $1.1763 \mathrm{E}-04$ & $1.1493 \mathrm{E}-04$ \\
\cline { 2 - 9 } & 1600 & $2.9516 \mathrm{E}-05$ & $2.8844 \mathrm{E}-05$ & $3.0085 \mathrm{E}-05$ & $2.8433 \mathrm{E}-05$ & $2.8615 \mathrm{E}-05$ & $3.0629 \mathrm{E}-05$ & $2.9902 \mathrm{E}-05$ \\
\hline \multirow{4}{*}{1.0} & 1800 & $1.1326 \mathrm{E}-05$ & $1.1097 \mathrm{E}-05$ & $1.1544 \mathrm{E}-05$ & $1.1093 \mathrm{E}-05$ & $1.1109 \mathrm{E}-05$ & $1.1528 \mathrm{E}-05$ & $1.1435 \mathrm{E}-05$ \\
\cline { 2 - 9 } & 1000 & $2.6698 \mathrm{E}-03$ & $2.6730 \mathrm{E}-03$ & $2.7090 \mathrm{E}-03$ & $2.6919 \mathrm{E}-03$ & $2.7266 \mathrm{E}-03$ & $2.5647 \mathrm{E}-03$ & $2.7324 \mathrm{E}-03$ \\
\cline { 2 - 9 } & 1200 & $1.3120 \mathrm{E}-04$ & $1.3018 \mathrm{E}-04$ & $1.3410 \mathrm{E}-04$ & $1.2904 \mathrm{E}-04$ & $1.3256 \mathrm{E}-04$ & $1.3810 \mathrm{E}-04$ & $1.3780 \mathrm{E}-04$ \\
\cline { 2 - 9 } & 1400 & $2.0887 \mathrm{E}-05$ & $2.0564 \mathrm{E}-05$ & $2.1491 \mathrm{E}-05$ & $1.9919 \mathrm{E}-05$ & $2.0514 \mathrm{E}-05$ & $2.2079 \mathrm{E}-05$ & $2.1861 \mathrm{E}-05$ \\
\cline { 2 - 9 } & 1600 & $6.5091 \mathrm{E}-06$ & $6.3648 \mathrm{E}-06$ & $6.6870 \mathrm{E}-06$ & $6.2408 \mathrm{E}-06$ & $6.3023 \mathrm{E}-06$ & $6.7916 \mathrm{E}-06$ & $6.6557 \mathrm{E}-06$ \\
\cline { 2 - 9 } & 1800 & $2.5486 \mathrm{E}-06$ & $2.4813 \mathrm{E}-06$ & $2.6088 \mathrm{E}-06$ & $2.4663 \mathrm{E}-06$ & $2.4610 \mathrm{E}-06$ & $2.6181 \mathrm{E}-06$ & $2.5700 \mathrm{E}-06$ \\
\hline
\end{tabular}

Table 2S. Adiabatic flame temperature of different fuels, unit: K.

\begin{tabular}{|c|c|c|c|c|c|c|c|c|}
\hline $\begin{array}{c}\text { Pressure } \\
(\mathbf{M P a})\end{array}$ & $\begin{array}{c}\text { Temperature } \\
\mathbf{( K )}\end{array}$ & $\mathbf{R P - 3}$ & $\mathbf{B F - 1}$ & $\mathbf{B F - 2}$ & $\mathbf{B F - 3}$ & $\mathbf{B F - 4}$ & $\mathbf{B F - 5}$ & BF-6 \\
\hline \multirow{3}{*}{0.1} & 1000 & 2781.42 & 2782.56 & 2781.44 & 2784.67 & 2782.87 & 2777.63 & 2779.32 \\
\cline { 2 - 9 } & 1400 & 2916.87 & 2916.30 & 2915.23 & 2918.03 & 2915.86 & 2914.01 & 2914.12 \\
\cline { 2 - 9 } & 1800 & 3032.47 & 3031.84 & 3030.79 & 3033.42 & 3031.19 & 3030.83 & 3030.50 \\
\hline \multirow{3}{*}{1.0} & 1000 & 2892.70 & 2896.03 & 2895.11 & 2899.19 & 2897.27 & 2886.74 & 2891.55 \\
\cline { 2 - 9 } & 1400 & 3104.83 & 3104.48 & 3103.40 & 3106.84 & 3104.33 & 3098.48 & 3100.69 \\
\hline
\end{tabular}

Table 3S. Burnout time of different fuels, unit: s.

\begin{tabular}{|c|c|c|c|c|c|c|c|c|}
\hline $\begin{array}{c}\text { Pressure } \\
(\mathbf{M P a})\end{array}$ & $\begin{array}{c}\text { Temperature } \\
(\mathbf{K})\end{array}$ & $\mathbf{R P - 3}$ & $\mathbf{B F - 1}$ & $\mathbf{B F - 2}$ & $\mathbf{B F - 3}$ & $\mathbf{B F - 4}$ & $\mathbf{B F - 5}$ & $\mathbf{B F}-6$ \\
\hline \multirow{3}{*}{0.1} & 1000 & 0.0191 & 0.0111 & 0.0205 & 0.0209 & 0.0202 & 0.0214 & 0.021 \\
\cline { 2 - 8 } & 1400 & 0.00179 & 0.00176 & 0.00182 & 0.00176 & 0.00207 & 0.00202 & 0.00161 \\
\cline { 2 - 9 } & 1800 & 0.00104 & 0.000679 & 0.000942 & 0.000997 & 0.000634 & 0.0011 & 0.000676 \\
\hline \multirow{2}{*}{1.0} & 1000 & 0.00289 & 0.00289 & 0.00297 & 0.00287 & 0.00295 & 0.00276 & 0.00297 \\
\cline { 2 - 9 } & 1400 & 0.0000945 & 0.0000951 & 0.0000856 & 0.0000894 & 0.0000867 & 0.000104 & 0.000103 \\
\hline
\end{tabular}

Table 4S. Extinction temperature of different fuels, unit: K.

\begin{tabular}{|c|c|c|c|c|c|c|c|c|}
\hline $\begin{array}{c}\text { Pressure } \\
(\mathbf{M P a})\end{array}$ & $\begin{array}{c}\text { Temperature } \\
\mathbf{( K )}\end{array}$ & $\mathbf{R P - 3}$ & $\mathbf{B F - 1}$ & $\mathbf{B F - 2}$ & $\mathbf{B F - 3}$ & $\mathbf{B F - 4}$ & BF-5 & BF-6 \\
\hline 0.1 & 298 & 1719.78 & 1723.07 & 1711.45 & 1714.67 & 1724.32 & 1715.38 & 1720.48 \\
\hline
\end{tabular}




\begin{tabular}{|l|l|l|l|l|l|l|l|l|}
1.0 & 298 & 1829.97 & 1841.57 & 1831.38 & 1830.78 & 1828.12 & 1833.03 & 1830.69 \\
\hline
\end{tabular}

Table 5S. Rate of production of different fuels, unit: $\mathrm{mol} \cdot \mathrm{cm}^{-3} \cdot \mathrm{s}^{-1}$.

\begin{tabular}{|c|c|c|c|c|c|c|c|c|}
\hline $\begin{array}{c}\text { Pressure } \\
(\mathbf{M P a})\end{array}$ & $\begin{array}{c}\text { Temperature } \\
(\mathbf{K})\end{array}$ & $\mathbf{R P - 3}$ & $\mathbf{B F - 1}$ & $\mathbf{B F - 2}$ & $\mathbf{B F - 3}$ & $\mathbf{B F}-4$ & BF-5 & BF-6 \\
\hline \multirow{3}{*}{0.1} & 1000 & 0.0857 & 0.0827 & 0.0821 & 0.0820 & 0.0824 & 0.0816 & 0.0824 \\
\cline { 2 - 9 } & 1400 & 0.0989 & 0.0988 & 0.1000 & 0.0998 & 0.0995 & 0.0995 & 0.0999 \\
\cline { 2 - 9 } & 1800 & 0.1215 & 0.1206 & 0.1198 & 0.1196 & 0.1200 & 0.1187 & 0.1198 \\
\hline \multirow{3}{*}{1.0} & 1000 & 5.0304 & 4.8118 & 4.7406 & 4.7835 & 4.8025 & 4.7456 & 4.7870 \\
\cline { 2 - 9 } & 1400 & 7.9416 & 7.7704 & 7.7120 & 7.6991 & 7.7481 & 7.6838 & 7.7431 \\
\cline { 2 - 9 } & 1800 & 10.4806 & 10.3838 & 10.3097 & 10.3120 & 10.3418 & 10.2314 & 10.3346 \\
\hline
\end{tabular}

Table 6S. Laminar flame speed of different fuels, unit: $\mathrm{cm} \cdot \mathrm{s}^{-1}$.

\begin{tabular}{|c|c|c|c|c|c|c|c|c|}
\hline $\begin{array}{c}\text { Pressure } \\
(\mathbf{M P a})\end{array}$ & $\begin{array}{c}\text { Temperature } \\
(\mathbf{K})\end{array}$ & $\mathbf{R P - 3}$ & $\mathbf{B F - 1}$ & $\mathbf{B F - 2}$ & $\mathbf{B F - 3}$ & $\mathbf{B F - 4}$ & BF-5 & BF-6 \\
\hline \multirow{3}{*}{0.1} & 300 & 39.56531 & 40.02115 & 39.59483 & 40.3372 & 39.84221 & 39.92278 & 39.80061 \\
\cline { 2 - 9 } & 400 & 60.44639 & 61.16298 & 60.45027 & 61.40544 & 60.99855 & 60.67646 & 60.69914 \\
\cline { 2 - 9 } & 500 & 88.36246 & 89.40058 & 88.39754 & 89.77763 & 89.12328 & 88.74625 & 88.71939 \\
\hline \multirow{3}{*}{1.0} & 600 & 123.3723 & 124.8146 & 123.4588 & 125.7953 & 124.4479 & 124.0051 & 123.8616 \\
\cline { 2 - 9 } & 300 & 19.60313 & 19.88826 & 19.501 & 20.04869 & 19.82706 & 19.65915 & 19.55454 \\
\cline { 2 - 9 } & 400 & 31.55536 & 31.90996 & 31.4102 & 32.38243 & 31.94016 & 31.63861 & 31.53963 \\
\cline { 2 - 9 } & 600 & 47.74994 & 48.26596 & 47.55626 & 48.74692 & 48.29026 & 47.81815 & 47.68149 \\
\hline
\end{tabular}

Table 7S. Extinction strain rate of different fuels, unit: $\mathrm{s}^{-1}$.

\begin{tabular}{|c|c|c|c|c|c|c|c|c|}
\hline $\begin{array}{c}\text { Pressure } \\
\text { (MPa) }\end{array}$ & $\begin{array}{c}\text { Temperature } \\
(\mathbf{K})\end{array}$ & RP-3 & BF-1 & BF-2 & BF-3 & BF-4 & BF-5 & BF-6 \\
\hline 0.1 & 800 & 402.8366 & 422.0438 & 418.585 & 424.3141 & 418.3768 & 418.3079 & 422.8051 \\
\hline 1.0 & 800 & 50.9124 & 56.68727 & 56.55461 & 56.6375 & 58.58655 & 56.13952 & 56.12944 \\
\hline
\end{tabular}

\section{CALCULATION RESULTS of GASOLINE and $\mathrm{H}_{2}$ /GASOLINE.}

Calculations of gasoline and $\mathrm{H}_{2} /$ gasoline (molar ratio 1:5) are conducted in an operating range of 1.0 at an equivalence ratio between 0.1 and $1.0 \mathrm{MPa}$, a temperature range from 300 to $1800 \mathrm{~K}$ based on a reduced surrogate mechanism of gasoline developed by Lawrence Livermore National Laboratory 3,4 . 
Table 8S. Ignition delay time of gasoline and $\mathrm{H}_{2}$ /gasoline, unit: $\mathrm{s}$.

\begin{tabular}{|c|c|c|c|}
\hline $\begin{array}{c}\text { Pressure } \\
(\mathbf{M P a})\end{array}$ & $\begin{array}{c}\text { Temperature } \\
(\mathbf{K})\end{array}$ & Gasoline & $\mathbf{H}_{\mathbf{2}} /$ Gasoline \\
\hline \multirow{4}{*}{0.1} & 1000 & $1.260 \mathrm{E}-02$ & $1.130 \mathrm{E}-03$ \\
\cline { 2 - 4 } & 1200 & $8.595 \mathrm{E}-04$ & $8.489 \mathrm{E}-05$ \\
\cline { 2 - 4 } & 1400 & $1.299 \mathrm{E}-04$ & $1.441 \mathrm{E}-05$ \\
\cline { 2 - 4 } & 1600 & $2.205 \mathrm{E}-05$ & $3.628 \mathrm{E}-06$ \\
\hline \multirow{5}{*}{1.0} & 1800 & $5.568 \mathrm{E}-06$ & $1.050 \mathrm{E}-06$ \\
\cline { 2 - 4 } & 1000 & $1.130 \mathrm{E}-03$ & $1.120 \mathrm{E}-03$ \\
\cline { 2 - 4 } & 1200 & $8.489 \mathrm{E}-05$ & $8.322 \mathrm{E}-05$ \\
\cline { 2 - 4 } & 1400 & $1.441 \mathrm{E}-05$ & $1.390 \mathrm{E}-05$ \\
\cline { 2 - 4 } & 1600 & $3.628 \mathrm{E}-06$ & $3.454 \mathrm{E}-06$ \\
\hline & 1800 & $1.050 \mathrm{E}-06$ & $9.994 \mathrm{E}-07$ \\
\hline
\end{tabular}

Table 9S. Adiabatic flame temperature of gasoline and $\mathrm{H}_{2} /$ gasoline, unit: $\mathrm{K}$.

\begin{tabular}{|c|c|c|c|}
\hline $\begin{array}{c}\text { Pressure } \\
\text { (MPa) }\end{array}$ & $\begin{array}{c}\text { Temperature } \\
(\mathbf{K})\end{array}$ & Gasoline & $\mathbf{H}_{\mathbf{2}} /$ Gasoline \\
\hline \multirow{3}{*}{0.1} & 1000 & 3141.182 & 3140.617 \\
\cline { 2 - 4 } & 1400 & 3196.203 & 3195.548 \\
\cline { 2 - 4 } & 1800 & 3251.370 & 3250.584 \\
\hline \multirow{3}{*}{1.0} & 1000 & 3466.878 & 3466.276 \\
\cline { 2 - 4 } & 1400 & 3540.486 & 3539.813 \\
\cline { 2 - 4 } & 1800 & 3614.702 & 3613.902 \\
\hline
\end{tabular}

Table 10S. Burnout time of gasoline and $\mathrm{H}_{2}$ /gasoline, unit: $\mathrm{s}$.

\begin{tabular}{|c|c|c|c|}
\hline $\begin{array}{c}\text { Pressure } \\
(\mathbf{M P a})\end{array}$ & $\begin{array}{c}\text { Temperature } \\
(\mathbf{K})\end{array}$ & Gasoline & $\mathbf{H}_{\mathbf{2}} /$ Gasoline \\
\hline \multirow{3}{*}{0.1} & 1000 & $6.745 \mathrm{E}-02$ & $7.714 \mathrm{E}-02$ \\
\cline { 2 - 4 } & 1400 & $2.622 \mathrm{E}-02$ & $2.777 \mathrm{E}-02$ \\
\cline { 2 - 4 } & 1800 & $2.068 \mathrm{E}-02$ & $1.520 \mathrm{E}-02$ \\
\hline \multirow{3}{*}{1.0} & 1000 & $4.309 \mathrm{E}-03$ & $4.487 \mathrm{E}-03$ \\
\cline { 2 - 4 } & 1400 & $1.692 \mathrm{E}-03$ & $1.564 \mathrm{E}-03$ \\
\cline { 2 - 4 } & 1800 & $1.401 \mathrm{E}-03$ & $1.195 \mathrm{E}-03$ \\
\hline
\end{tabular}

Table 11S. Extinction temperature of gasoline and $\mathrm{H}_{2} /$ gasoline, unit: $\mathrm{K}$.

\begin{tabular}{|c|c|c|c|}
\hline $\begin{array}{c}\text { Pressure } \\
(\mathbf{M P a})\end{array}$ & $\begin{array}{c}\text { Temperature } \\
(\mathbf{K})\end{array}$ & Gasoline & $\mathbf{H}_{\mathbf{2}} /$ Gasoline \\
\hline 0.1 & 298 & 1676.185 & 1673.489 \\
\hline 1.0 & 298 & 1842.189 & 1840.554 \\
\hline
\end{tabular}


Table 12S. Rate of production of gasoline and $\mathrm{H}_{2} /$ gasoline, unit: $\mathrm{mol} \cdot \mathrm{cm}^{-3} \cdot \mathrm{s}^{-1}$.

\begin{tabular}{|c|c|c|c|}
\hline $\begin{array}{c}\text { Pressure } \\
(\mathbf{M P a})\end{array}$ & $\begin{array}{c}\text { Temperature } \\
(\mathbf{K})\end{array}$ & Gasoline & $\mathbf{H}_{\mathbf{2}} /$ Gasoline \\
\hline \multirow{3}{*}{0.1} & 1000 & 0.348 & 0.357 \\
\cline { 2 - 4 } & 1400 & 0.451 & 0.462 \\
\cline { 2 - 4 } & 1800 & 0.583 & 0.595 \\
\hline \multirow{3}{*}{1.0} & 1000 & 29.922 & 30.536 \\
\cline { 2 - 4 } & 1400 & 39.662 & 40.475 \\
\cline { 2 - 4 } & 1800 & 51.792 & 52.863 \\
\hline
\end{tabular}

Table 13S. Laminar flame speed of gasoline and $\mathrm{H}_{2} /$ gasoline, unit: $\mathrm{cm} \cdot \mathrm{s}^{-1}$.

\begin{tabular}{|c|c|c|c|}
\hline $\begin{array}{c}\text { Pressure } \\
\text { (MPa) }\end{array}$ & $\begin{array}{c}\text { Temperature } \\
(\mathbf{K})\end{array}$ & Gasoline & $\mathbf{H}_{2} /$ Gasoline \\
\hline \multirow{3}{*}{0.1} & 300 & 44.103 & 44.805 \\
\cline { 2 - 4 } & 400 & 66.977 & 68.485 \\
\cline { 2 - 4 } & 500 & 97.189 & 99.411 \\
\cline { 2 - 4 } & 600 & 135.909 & 138.201 \\
\hline \multirow{4}{*}{1.0} & 300 & 21.160 & 21.618 \\
\cline { 2 - 4 } & 400 & 34.049 & 34.764 \\
\cline { 2 - 4 } & 500 & 51.572 & 52.563 \\
\cline { 2 - 4 } & 600 & 73.942 & 75.678 \\
\hline
\end{tabular}

Table 14S. Extinction strain rate of gasoline and $\mathrm{H}_{2} /$ gasoline, unit: $\mathrm{s}^{-1}$.

\begin{tabular}{|c|c|c|c|}
\hline $\begin{array}{c}\text { Pressure } \\
(\mathbf{M P a})\end{array}$ & $\begin{array}{c}\text { Temperature } \\
(\mathbf{K})\end{array}$ & Gasoline & $\mathbf{H}_{\mathbf{2}} /$ Gasoline \\
\hline 0.1 & 800 & 189.8593 & 200.4471 \\
\hline 1 & 800 & 84.6809 & 88.5887 \\
\hline
\end{tabular}

Table 15S. Disturbance of $\mathrm{H}_{2}$ to gasoline and RP-3 aviation kerosene.

\begin{tabular}{|c|c|c|}
\hline Disturbance & gasoline & $\mathbf{R P - 3}$ \\
\hline$d_{1}$ (Ignition Delay Time) & 2.91 & 2.56 \\
\hline $\begin{array}{c}d_{2} \text { (Adiabatic Flame } \\
\text { Temperature) }\end{array}$ & 1.06 & 0.9 \\
\hline$d_{3}$ (Burnout Time) & 1.37 & 2.31 \\
\hline$d_{4}$ (Extinction Temperature) & 0.35 & 0.79 \\
\hline$d_{5}$ (Rate of Production) & 1.12 & 1.18 \\
\hline$d_{6}$ (Laminar Flame Speed) & 0.54 & 0.56 \\
\hline$d_{7}$ (Extinction Strain Rate) & 0.65 & 0.76 \\
\hline $\boldsymbol{D}$ & $\mathbf{7 . 9 9}$ & $\mathbf{9 . 0 6}$ \\
\hline
\end{tabular}




\section{REFERENCES}

[1] ANSYS Chemkin 17.0 (15151), ANSYS Reaction Design: San Diego, 2016.

[2] Egolfopoulos, F. N.; Campbell, C. S. Unsteady counterflowing strained diffusion flames: diffusion-limited frequency response. Journal of Fluid Mechanics 1996, 318, 1-29.

[3] Mehl, M.; Pitz, W. J.; Westbrook, C. K.; Curran, H. J. Kinetic modeling of gasoline surrogate components and mixtures under engine conditions. Proceedings of the Combustion Institute 2011, 33, 193-200.

[4] Mehl, M.; Chen, J. Y.; Pitz, W. J.; Sarathy, S. M., Westbrook, C. K. An approach for formulating surrogates for gasoline with application toward a reduced surrogate mechanism for CFD engine modeling. Energy Fuels 2011, 25, 5215-5223. 